\title{
A Dynamic Optimization for Local Bus Systems
}

\author{
Yibing Wang ${ }^{1}$, Yuqing Tian ${ }^{1,2}$ \\ ${ }^{1}$ School of Business, Shandong University at Weihai, Weihai, 264209, China \\ ${ }^{2}$ School of Translation Studies, Shandong University at Weihai, Weihai, 264209, China
}

Keywords: schedule, optimization, bus system

\begin{abstract}
This paper develops a dynamic model to solve the vehicle schedule of a real multi-route bus system at the city Weihai of China. The model allows for the interactions of potential passengers and buses so as to achieve the maximum profit and passenger satisfaction. The model generated satisfying results in terms of financial benefits and social welfare.
\end{abstract}

\section{Introduction}

The traffic volume is increasing dramatically along with the increase of private vehicles in most cities in the world. Making effective use of public transportation, such as bus systems, becomes a top priority to many cities. Bus systems play a significant role in many cities. From the view point of the city, successful bus companies bring revenue to the community as well as employment. Moreover a bus system provides mobility for a wide variety of individuals that may not have access to a private vehicle for health or economic reasons. In order to achieve the potential service, economic and environmental advantages it is important that the city administration and bus companies design routes and schedules considering customers' response. A scientific approach to these decisions can improve the system's societal value.

In this paper, we investigate the problem of how buses should be allocated to routes: for a given bus system with known demand, costs and routes, the objective is to determine the headways for each route at each time period that minimizes the cost. The number of buses allocated to the routes will also be found when the headway is determined, based on a maximum utilization principle.

\section{Literature review}

The bus scheduling problem, arising in public transport bus companies, addresses the task of assigning buses to cover a given set of timetabled trips with consideration of practical requirements. Bunte and Kliewer (2009) produced an overview and discussed the modeling approaches for different kinds of vehicle scheduling problems and gave an up-to-date comprehensive overview on the basis of a general problem definition [1]. And he defined the Vehicle Scheduling Problem (VSP).

Under most circumstances, the objective of VSP model was to minimize cost. The cost could be operation cost which was from gas, repairing or setup cost like drivers' salary. Salzborn and Franz (1972) pointed out that typically the cost could be simplified as the fleet size of a transportation system. Actually this approach to optimum bus scheduling represented one typical theory that vehicle movement was thought of as continuous time dependent flows on the links of a network [2].

A similar but more intuitive case was described in Kliewer (2006) in which the author discussed the multi-depot, multi-vehicle bus scheduling problem (MDVSP) involving multiple depots for vehicles and different vehicle types for timetabled trips and used a time-space-based model instead of connection-based networks for MDVSP modeling. This lead to a crucial reduction in the size of the corresponding mathematical models compared to well-known connection-based network flow or set partitioning models [3]. Naumann and Kramkowski (2011) defined waiting time and delay penalty on arcs in a Time-Space Network (TSN) in a new stochastic programming approach for robust vehicle scheduling for public bus transport [4]. Luethi (2007) made direct contribution to the modeling in this paper. His research evaluated the influence of headway and other factors on 
passenger arrival rates at public transport stations based on data collected at 28 stations in Zurich's public transport network [5].

Customer demand and preference for urban public transportation have also been investigated by many researchers. Microscopically Strathman (2003) made a research on the effects of headway deviation on bus passenger loads based on data of Tri-Met, the public transit system in the Portland area and drew an important conclusion that in peak hours a delay for 1 minute of a bus led to an increase of 2.6 to the load of a bus, which means in the situation that he investigated, the passenger's arrival rate was 2.6 and this is the most direct reference on passenger's deterministic arrival rate [6]. Macroscopically Frankena and Mark (1978) empirically estimated the demand functions for urban bus services in Canada. Based on this he concluded that the quantity of bus service demanded per capita in an urban area depended upon the money and time costs of travel by bus and by average income and other socioeconomic characteristics of the population and geographical characteristics of the urban area [7]. Paulley et al (2006) also investigated the factors affecting the demand for public transport. While a wide range of factors were examined in the study, the paper concentrated on the findings regarding the influence of fares, quality of service and income and car ownership. He drew a series of interesting conclusions like public transport use was remarkably sensitive to car costs but car use was much less dependent on public transport costs, and the effect of service quality was much less than that of fares. Besides, income and car ownership growth were fundamental to the underlying demand for public transport and there had been almost continual decline in the demand for bus travel over the past 25 years [8]. Golob (1972) discussed the structure of a market research study to design an evolutionary public transportation system [9]. These scholars' work helped more on macroscopic research on public transportation planning issues.

Comprehensively, the bus scheduling problem was usually included as a part of urban transportation planning research and analysis, along with vehicle routing problem (VRP) [10]. A typical example was the book written by Meyerand Miller (2001) that held the opinion that the major purpose of transportation planning was to inform decision making. The book of Domencich and McFadden (1975) developed a theory of demand, for populations of individual economic consumers [11].

Researchers used to regard passengers and bus companies as isolated factors and they analyzed and made decisions unilaterally ignoring the impact of their decisions on the other agent. Hardly any researchers considered prospective passengers and decision makers of bus companies as interactive factors, however the world is connected and any change may lead to unexpected feedback. In this paper, the intent is to reflect this characteristic in a simple and computationally feasible way that will allow investigation of alternatives.

\section{Problem description}

For any single bus route, the frequency of buses affects the time that passengers wait at bus stations in a logarithmic rate [5]. Furthermore it affects the tendency that passengers will take a bus to their destination to some degree. Ben-Akiva (2002) made comparisons between customer choice on buses and railways and revealed that lower frequency did harm the interest of taking any public transportation [12]. Therefore it was supposed that the demand for a bus route was a function of its headway [13].

For the bus company, higher route frequency brings more passengers, however, higher cost as well. The tradeoff between losing passengers and reducing cost could reach a balance and they all can be formed into an optimization problem. In addition, since such decisions are made for the common good, social welfare, environmental concerns and other externalities may be important to consider.

We begin by setting up some basic concepts that are then applied to models of general scope. Consider Figure 1 showing a basic route consisting of a sequence of bus stops. A bus starts every Trt time. We assume spacing remains constant. The route length is $\mathrm{Lr}$ and the travel speed is vrt. Initially assume the bus retraces its steps - "out of service" after each run and travels at the same speed. To reach the most utilization of a fixed number of buses so as to minimize rider wait time 
and maximize route rider capacity, when the backward travelling bus arrives at the start station this bus should be dispatched immediately to begin the next trip. This maintains the bus separation as Trt in time or Lr/vrt in distance. In this deterministic model arrival time between consecutive buses at a stop is always Trt time units.

Therefore with no delay at the start and end stations, since the number of buses is equal to the time for a tour (2Lr/vrt) times the tour completion rate (1/Trt), we obtain (1).

$$
M_{r t}=2 L_{r} / v_{r t} T_{r t}
$$

To explain the situation in a more intuitive angle, the time that a bus spends on the service portion of the route equals the summation of the spacing between half of the buses, which leads to (2), the basic equation of the Maximum Utilization Principle in this case.

$$
L_{r} / v_{r t}=M_{r t} T_{r t} / 2
$$

Actually (1) is the transformed Little's Law for this case. Little's Law which is commonly expressed as $L=\lambda W$, defines the mean relationship between parameters in steady-state queuing systems. For a stable system, the average number of entities $L$ equals to the average entity arrival rate, $\lambda$, times the average time an entity spends in the system, $W$. Referring to (1), $L$ is substituted by $M_{r t}, \lambda$ is substituted by $\left(1 / T_{r t}\right)$, and $W$ is substituted by $\left(2 L_{r} / v_{r t}\right)$.

Furthermore the Maximum Utilization Principle can be released to inequality since the idealized case may not be necessary to achieve. Then the principle can be generalized to enable the model to reflect any bus route cases besides the simplest case like Figure 1.

\section{Model and results}

Weihai Bus Transportation serves Weihai of Shandong Province over 30 bus routes. Annual ridership is over 50 million people. This transportation system is a reliable resource of research and provides a basis for examining bus operations.

A realistic scenario is that the day is divided into two scenarios according to whether it was busy hours or not. Furthermore, it was common that several bus routes shared a number of buses which were dispatched to meet their respective demand. In this case, Route K1 and Route K2 were taken into consideration along with Route 12 since they shared the Wehai Transportation Center as a starting or ending station and it was possible to make the bus dispatching flexible. A model was built to come up with a comprehensive optimal solution for the chosen routes.

The ridership data was collected from the Weihai Bus Transportation Bureau and the route length was tested by Baidu Map. The artificial parameters were derived from methods of [5] upon observations on these routes. The total number of buses was limited to 20 . Then we have the model as follows:

$$
\begin{array}{ll}
\text { Max. } U=b \sum_{t} \sum_{r} N_{t r}-\sum_{t} \sum_{r} c_{r} P_{r t} / T_{r t} & \text { (3) } N_{r t}=\left(1.64-0.21 \ln T_{r t}\right) N_{r t o} \\
\left(L_{r}+\xi v_{r t}\right) / v_{r t} T_{r t} \leq M_{r t} & \text { (5) } \sum_{r} M_{r} \leq M_{0} \\
1.45 \log \left(T_{r t}\right) \leq \varepsilon & \text { (7) }\left(\gamma s_{r} P_{r t} / T_{r t}\right) / N_{r t} \geq \sigma \\
f\left(L_{r}+\xi v_{r t}\right) / v_{r t} T_{r t} \geq \delta & \text { (9) }
\end{array}
$$

In the model $M_{0}$ is the total number of buses distributed to the selected routes by the operating bus company. $T_{r t}$ is the headway of buses on route $r$ in time $t . L_{r}$ represents the length of route $r$. $M_{r t}$ stands for the total number of buses on route $r$ at time period $t$. $v_{r t}$ is an average bus travel speed at route $r$ in time $t . P_{t}$ is the length of time period of scenario $t . c_{r}$ is the unit cost for one trip of buses of route r, for all $t . s_{r}$ is the Number of seats of a bus on route $r . N_{r t}$ is the ridership (potential demand) of route $r$ in scenario $t$. $\mathrm{b}$ is the average fare of one bus rider.

The model was built in AMPL and used the MINOS solver, a linear and nonlinear mathematical optimization solver. The most significant characteristic of AMPL is that it enables users to describe complex mathematical models by simple algebraic symbols that match with the describing and thinking method of modellers. 
And the results are given in Table 1. A series of graphs can be generated and visualize how the profit and expected passengers waiting time are influenced by the number of available buses (See Figure 1 and Figure 2). In Figure 2 the AWT is the average waiting time of all three bus routes.

It can be observed from the sensitivity analysis that $M$ provides a significant condition for the model as well as operations in reality and increasing of $\mathrm{M}$ brings more benefit to the system. On the other hand Figure 1 and 2 reveals that there should be an upper limit for $\mathrm{M}$ and beyond which the ridership and profit can hardly been improved further. Moreover, increasing $\mathrm{M}$ may also bring new financial burden and such decision requires more consideration and discussion.

\section{Conclusion}

This paper developed a new optimization model for a bus assignment problem that took the interaction between customers and system cost into consideration. By providing advice on bus headway, the model may be viewed as an integrated, strategic input to the more widely researched vehicle scheduling problem that requires the bus timetable as input. The optimization model generated satisfying results for situation that multiple routes and time periods were investigated. Since the calculation result was quite reasonable, several highlights could be concluded.

First, the model has an objective function requiring only minimal data - bus costs per route trip and set of decision periods. Only three constraints were always necessary. The total number of constraints depends on the complexity of scenarios. In real operation process the operators could divide the system to maintain the complexity of subsystems in a relatively low degree and apply the model to help scheduling. In general the model is concise enough that it presented little difficulty in solving with standard optimization engines, at least for small city examples.

Second, currently the local buses of Weihai Huancui District operate with headway between 20 to 30 minutes. The suggested headway given by the model in this paper varies from over 10 minutes to over 30 minutes. Literally the difference between current operating pattern and newly suggested one was not so large that adjustment on bus schedule would be relatively difficult to implement. Besides the calculation result of the optimization model could also help on bus dispatching that could promote the utilization of the resources that the bus company was able to govern.

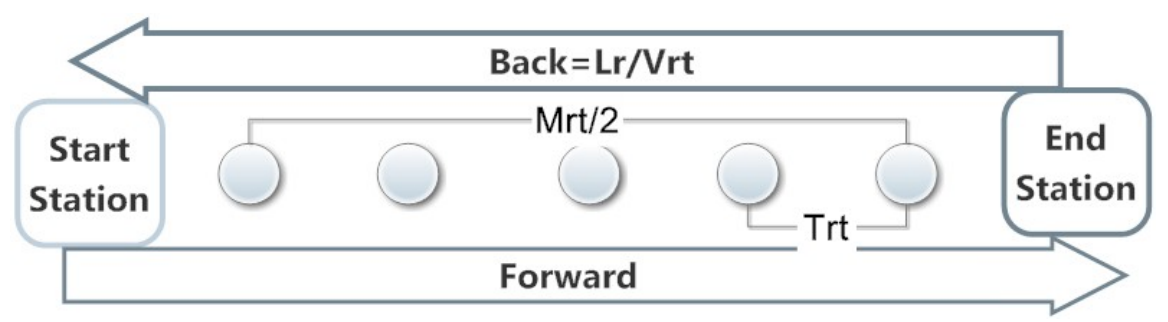

Fig.1: A basic route

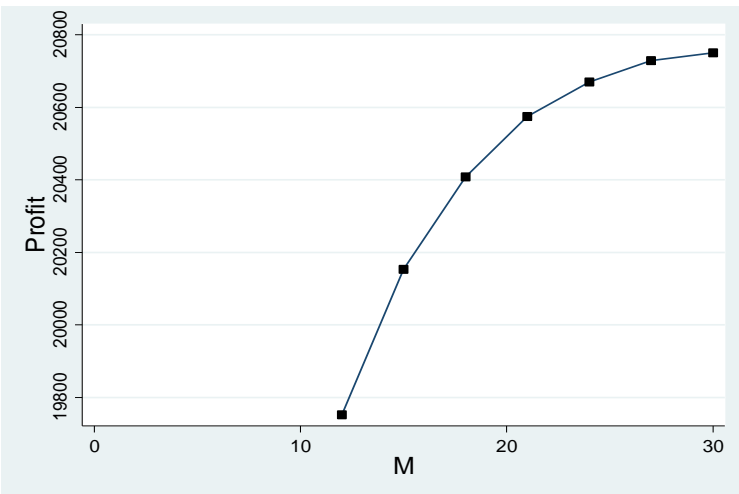

Fig.2: $\mathrm{M}$ vs. profit

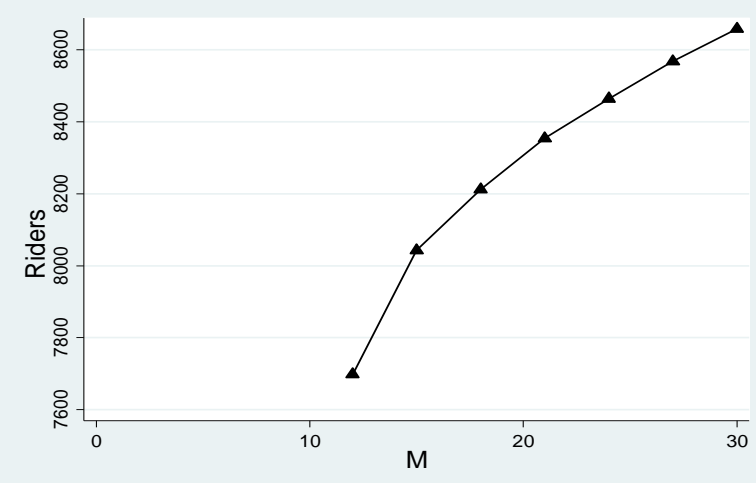

Fig.3: M vs. ridership 
Table 1: Bus schedule solution

\begin{tabular}{ccccc}
\hline & \multicolumn{2}{c}{ Busy Hours } & \multicolumn{2}{c}{ Non-Busy Hours } \\
\cline { 2 - 5 } Route & $\begin{array}{l}\text { Headway } \\
\text { (Minutes) }\end{array}$ & Number of Buses & $\begin{array}{l}\text { Headway } \\
\text { (Minutes) }\end{array}$ & Number of Buses \\
\hline 12 & 19.0 & 8 & 21.5 & 5 \\
K1 & 8.5 & 6 & 10.5 & 4 \\
K2 & 12.5 & 6 & 15.5 & 4 \\
\hline
\end{tabular}

Table 2: Sensitivity analysis on $\mathrm{M}$

\begin{tabular}{cccccc}
\hline $\mathbf{M}$ & $\begin{array}{c}\text { Profit } \\
\text { (¥) }\end{array}$ & Routes & Tr1/Tr2 & $\begin{array}{c}\text { N(Tr1)+N(Tr2) } \\
\text { (Total Riders) }\end{array}$ & WTw \\
\hline 3 & - & - & - & - & - \\
6 & - & - & - & - & - \\
9 & - & - & - & - & - \\
& & 12 & $28.2 / 25.6$ & 2984 & 4.3 \\
12 & 19752 & K1 & $17.8 / 12.6$ & 2451 & 4.8 \\
& & K2 & $26.3 / 18.8$ & 2263 & 4.3 \\
& & 12 & $26.9 / 21.7$ & 3059 & 4.7 \\
15 & 20154 & K1 & $12.0 / 10.5$ & 2588 & 3.6 \\
& & K2 & $17.8 / 15.8$ & 2396 & 4.2 \\
& & 12 & $21.6 / 21.7$ & 3124 & 4.6 \\
18 & 20408 & K1 & $9.6 / 10.5$ & 2640 & 3.4 \\
& & K2 & $14.3 / 15.8$ & 2448 & 4.0 \\
& & 12 & $18.1 / 21.7$ & 3179 & 4.4 \\
21 & 20575 & K1 & $8.0 / 10.5$ & 2684 & 3.3 \\
& & K2 & $11.9 / 15.8$ & 2491 & 3.9 \\
& & 12 & $15.7 / 21.7$ & 3221 & 4.3 \\
24 & 20670 & K1 & $6.9 / 10.5$ & 2718 & 3.2 \\
& & K2 & $10.3 / 15.8$ & 2525 & 1.8 \\
& & 12 & $13.8 / 21.7$ & 3260 & 4.2 \\
27 & 20729 & K1 & $6.0 / 10.5$ & 2751 & 3.1 \\
& & K2 & $8.9 / 15.8$ & 2557 & 3.7 \\
& & 12 & $12.3 / 21.7$ & 3294 & 4.2 \\
30 & 20751 & K1 & $5.3 / 10.5$ & 2779 & 3.0 \\
& & K2 & $7.9 / 15.8$ & 2585 & 3.6 \\
\hline
\end{tabular}

\section{Acknowledgements}

This work is supported by Shandong Natural Science Foundation (SNSF) of China under Grant ZR2014GM004.

\section{References}

[1] Bunte, Stefan, and Natalia Kliewer. An overview on vehicle scheduling models. Public Transport, 1.4, pp. 299-317, 2009

[2] Salzborn, Franz JM. Optimum bus scheduling. Transportation Science, 6(2), pp.137-148, 1972 
[3] Kliewer, Natalia, Taieb Mellouli, and Leena Suhl. A time-space network based exact optimization model for multi-depot bus scheduling. European journal of operational research, 175(3), pp. 1616-1627, 2006

[4] Naumann, Marc, Leena Suhl, and Stefan Kramkowski. A stochastic programming approach for robust vehicle scheduling in public bus transport. Procedia-Social and Behavioral Sciences, 20(1), pp. 826-835, 2011

[5] Luethi, Marco, Ulrich Alois Weidmann, and Andrew Nash. Passenger arrival rates at public transport stations. Transportation Research Board 86th Annual Meeting. No. 07-0635. 2007.

[6] Strathman, James G., Thomas Jeffrey Kimpel, and Steve Callas. Headway deviation effects on bus passenger loads: Analysis of Tri-Met's archived AVL-APC Data. No. TNW2003-01, Transportation Northwest, Department of Civil Engineering, University of Washington, 2003.

[7] Frankena, Mark W. The demand for urban bus transit in Canada. Journal of Transport Economics and Policy, 3(1), pp. 280-303, 1978

[8] Paulley, Neil, Richard Balcombe, Roger Mackett, Helena Titheridge, John Preston, Mark Wardman, Jeremy Shires and Peter White. The demand for public transport: The effects of fares, quality of service, income and car ownership. Transport Policy, 13(4), pp. 295-306, 2006

[9] Golob, Thomas F., Eugene T. Canty and Richard L. Gustafson. An analysis of consumer preferences for a public transportation system. Transportation Research, 6(1), pp. 81-102, 1972

[10]Fügenschuh, Armin. Solving a school bus scheduling problem with integer programming. European Journal of Operational Research, 193(3), pp.867-884, 2009

[11] Domencich, Thomas A., and Daniel McFadden. Urban Travel Demand-A Behavioral Analysis. Monograph, No 113. 1975.

[12] Ben-Akiva, Moshe, and Takayuki Morikawa. Comparing ridership attraction of rail and bus. Transport Policy, 9(2), pp.107-116, 2002

[13]Beirão, Gabriela, and J. A. Sarsfield Cabral. Understanding attitudes towards public transport and private car: A qualitative study. Transport policy, 14(6), pp. 478-489, 2007 